\title{
Abstract: Some Investigations on Robustness of Deep Learning in Limited Angle Tomography
}

\author{
Yixing Huang ${ }^{1}$, Tobias Würfl${ }^{1}$, Katharina Breininger ${ }^{1}$, Ling Liu ${ }^{1}$, \\ GÃ $\frac{1}{4}$ nter Lauritsch ${ }^{2}$, Andreas Maier ${ }^{1,3}$ \\ ${ }^{1}$ Friedrich-Alexander Universität Erlangen-Nürnberg, 91058 Erlangen, Germany \\ ${ }^{2}$ Siemens Healthcare GmbH, 91301 Forchheim, Germany \\ ${ }^{3}$ Erlangen Graduate School in Advanced Optical Technologies (SAOT), \\ 91058 Erlangen, Germany \\ yixing.yh .huang@fau.de
}

In computed tomography, image reconstruction from an insufficient angular range of projection data is called limited angle tomography. Due to missing data, reconstructed images suffer from artifacts, which cause boundary distortion, edge blurring, and intensity biases. Recently, deep learning methods have been applied very successfully to this problem in simulation studies. However, the robustness of neural networks for clinical applications is still a concern. It is reported that most neural networks are vulnerable to adversarial examples.

In this paper, we aim to investigate whether some perturbations or noise will mislead a neural network to fail to detect an existing lesion.

Our experiments demonstrate that the trained neural network, specifically the U-Net, is sensitive to Poisson noise. While the observed images appear artifact-free, anatomical structures may be located at wrong positions, e.g. the skin shifted by up to $1 \mathrm{~cm}$. This kind of behavior can be reduced by retraining on data with simulated Poisson noise. However, we demonstrate that the retrained U-Net model is still susceptible to adversarial examples. We conclude the paper with suggestions towards robust deep-learning-based reconstruction [1].

\section{References}

1. Huang Y, Würfl T, Breininger K, et al. Some investigations on robustness of deep learning in limited angle tomography. Proc MICCAI. 2018; p. 145-153. 